\title{
Tremor induced by toluene misuse successfully treated by a Vim thalamotomy
}

\author{
Yasushi Miyagi, Fumio Shima, Katsuya Ishido, Taeko Yasutake, Kazufumi Kamikaseda
}

\begin{abstract}
A 22 year old man developed a vigorous tremor of $5 \mathrm{~Hz}$ in his right hand, after a 7 year history of toluene misuse. T2 Weighted MRI depicted marked decreases in the signal intensity of the basal ganglia, red nucleus, and thalamus on both sides. The stereotactic coagulation of the left nucleus ventrointermedius (Vim) of the thalamus abolished the tremors in his right hand. This patient clearly exhibited the pathological involvement of rubral lesions in generation of a toluene induced tremor on MRI. Toluene induced tremor is an irreversible symptom which persists even after stopping toluene misuse, therefore in medically intractable cases, it should be positively treated by a Vim thalamotomy.

(F Neurol Neurosurg Psychiatry 1999;66:794-796)
\end{abstract}

Keywords: toluene misuse; Vim thalamotomy

Department of

Neurological Surgery

Y Miyagi

K Ishido

K Kamikaseda

Department of

Neurology, Kaizuka

Hospital, 7-7-27

Hakozaki, Japan

$\mathrm{T}$ Yasutake

Department of Clinical Neurophysiology, Neurological Institute,

Faculty of Medicine, Kyushu University,

3-1-1 Maidashi,

Higashi-Ku, Fukuoka

812, Japan

F Shima

Correspondence to: Dr Yasushi Miyagi,

Department of Neurological

Surgery, Kaizuka Hospital,

7-7-27 Hakozaki,

Higashi-Ku, Fukuoka 812,

Japan. Telephone 008192

632 3333; fax 008192632

2230 .

Received 21 August 1998 and in revised form

8 December 1998

Accepted 1 December 1998 though medication with isoproterenol and clonazepam helped to control the tremor to some degree for a year, the tremor in his right hand gradually progressed.

At admission, a vigorous postural tremor (5-6 $\mathrm{Hz}$ ) which increased with voluntary movements was seen in the patient's right hand. A tremor was also present but less intense in his left hand. No tremor was present at rest. As a result, the tremor was not considered to be associated with either cogwheel rigidity, pyramidal signs, or cerebellar dysfunction. He had a mild cognitive dysfunction (verbal IQ 82, motor IQ 60, general IQ 69) and a mild dysarthria. The findings on EEG and the auditory brainstem response were all normal. T1 Weighted MRI showed mild brain atrophy, whereas the T2 weighted images showed a marked decrease in the signal intensities of the basal ganglia, red nucleus, and thalamus on both sides (figure A). As the patient's tremor was slowly progressive, even after stopping toluene misuse, and the MRI suggested organic lesions bilaterally in the red nucleus, a stereotactic Vim thalamotomy was thus planned; such a procedure has been successful in treating other similar types of tremors. For the intraoperative estimation of the tremor, the patient stopped all medication 18 hours before surgery. The patient's head was initially fixed in a Leksell's stereotactic frame and he underwent $\mathrm{CT}$ and MRI to determine the coordinates of the anterior and posterior commissures (AC, PC). The localisation of the target in the left Vim of the thalamus was finally calculated from the AC-PC line. Under a local infiltration of xylocaine, a burr hole was opened on the frontal bone $3 \mathrm{~cm}$ left of the midline and $5 \mathrm{~mm}$ anterior to the coronal suture. Vim was localised $5.5 \mathrm{~mm}$ anterior to the PC and $14 \mathrm{~mm}$ left of the AC-PC line based on the CT and MRI findings. During the operative procedures, the postural tremor of the patient's right arm was recorded with EMG and any intentional tremors were noted by having the patient draw figures. Before the coagulation of the left Vim, the patient drew poorly because of the intentional tremor. As soon as the coagulation of the left Vim was started (figure B), the tremor in the right hand abruptly decreased and he was thereafter able to draw well (figure $\mathrm{C}$ ). At the end of the coagulation $\left(70^{\circ} \mathrm{C}, 60\right.$ minutes with a Leksell Neuro Generator), the postural and action tremor of the right hand were abolished and the grouped 

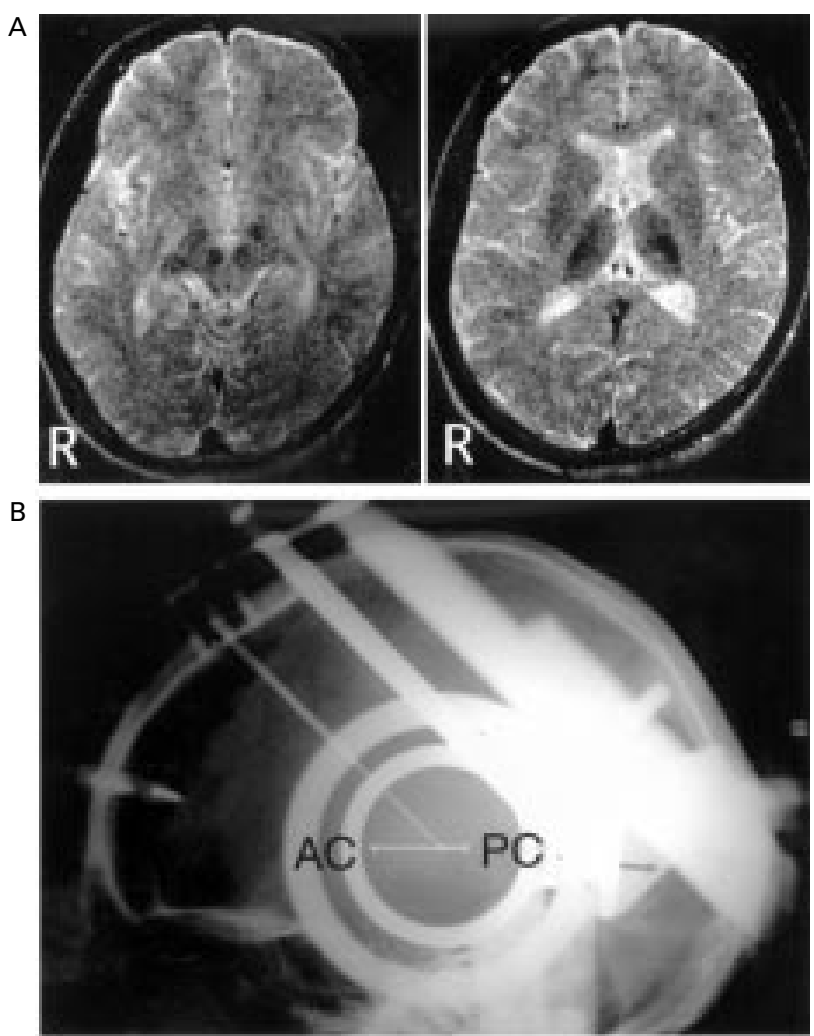

C

Before coagulation

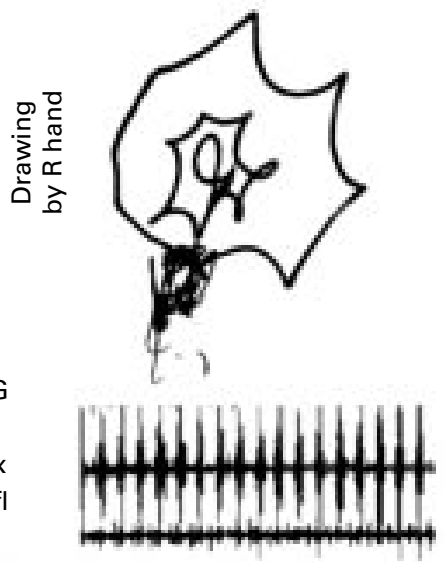

EMG

(A) T2 Weighted MRI (repetition time, $2000 \mathrm{~ms}$; echo time, $110 \mathrm{~ms}$ ) showing the low signal intensities of the thalamus, basal ganglia, and red nucleus on both sides. (B) Intraoperative plain skull film of a lateral projection showing the site of the coagulation electrode. The superimposed points indicate the anterior commissure $(A C)$ and posterior commissure (PC) as determined by CT. The tip of the coagulation electrode was located 5.5 $\mathrm{mm}$ anterior to the $P C$ and $14 \mathrm{~mm}$ left to the AC-PC line (Vim of the thalamus). (C) Intraoperative recording of an EMG and figure drawing by the patient with his right hand,before and after the coagulation of the left Vim.

discharges showing the tremor rhythm also disappeared on the EMG. He voiced no complaints during the operation, and his postoperative course was uneventful. He returned to work 1 week after the operation, and he is still free from tremors after a 2 year follow up period without medication.

\section{Discussion}

The symptoms related to the CNS found in cases of toluene misuse include cerebellar dysfunction, cognitive dysfunction, pyramidal signs, cranial nerve dysfunction, and involuntary movements. A wide range of clinical symptoms among such patients has been reported, probably due to differences in the duration of toluene inhalation or the type of organic thinners mixed with toluene. ${ }^{6}$

The clinical features of toluene induced tremors are as follows: (1) tremors are found mainly in the upper extremities, (2) they have a frequency of $4-5 \mathrm{~Hz}$, (3) postural or intentional tremors are present but there are no resting tremors, (4) the tremors are generally intractable to medical treatment. The pathogenesis of toluene induced tremors has been considered to involve lesions of the dentatorubro-olivary system, ${ }^{7}$ and a decrease in the signal intensity of the basal ganglia, thalamus, and tegmentum of the brainstem on the T2 weighted MR image has also been suggested to correlate with this lesion. ${ }^{6-8}$ Our patient also had symptoms which closely correlated with the typical clinical features and the marked decreases in the signal intensities of the bilateral thalamus and red nucleus on the T2 weighted MRI. Rubral tremors, which are often referred to as midbrain tremors, tend to exhibit a slow frequency $(2-3 \mathrm{~Hz})$ as well as a resting component. ${ }^{9}$ In our patient, the tremor was somewhat faster $(5-6 \mathrm{~Hz})$ than typical rubral tremors and it was also not seen at rest, which thus suggested that the toluene induced tremor in our patient was not only due to lesions in the red nucleus but was also influenced by multiple lesions in the basal ganglia and thalamus.

Experimentally, ventromedial tegmental lesions including damage to the red nucleus and cerebellothalamic pathway, tend to generate the tremor rhythms in the contralateral upper and lower limbs (synchronisation of neuronal discharge). A possible descending pathway that mediates the tremor rhythm seems to be the reticulospinal tract originating from the pontine tegmental reticular formation. The proprioceptive signal from the muscle spindle associated with tremors, in turn, ascends in the contralateral spinothalamic tract to the Vim. The signal probably projects to the cortical area $3 \mathrm{a}$, and then comes down again from the cortex. ${ }^{10}$ As a result, the thalamocortical circuit around the Vim seems to play an important part in the generation and maintenance of the tremor rhythm. In our case, the rubral lesion demonstrated by MRI clearly showed the pathological contribution of the ventromedial tegmental lesions to the generation of such tremors. The stereotactic Vim thalamotomy dramatically cured the tremor in our patient, which thus suggests the involvement of rubral lesions and the thalamocortical circuit in the development and/or maintenance of toluene induced tremors.

Clonazepam, but not $\beta$ blockers, sometimes attenuates both midbrain tremors ${ }^{11}$ and toluene induced tremors (including hyperkinesie volitionnelle), ${ }^{7}$ however, in most cases, a midbrain tremor is generally intractable to medical therapy. Our patient was also treated with clonazepam because a $\beta$ blocker had no effect. However, the tremors could not be completely controlled. There has been no 
previous report of a spontaneous resolution of toluene induced tremors. Although our patient had stopped misuse of toluene for more than a year before admission, his tremors nevertheless gradually deteriorated. Sodeyama et $a l^{7}$ described a patient who developed a tremor and hyperkinesie volitionnelle 3 years after stopping toluene misuse. Therefore, the involuntary movement due to toluene misuse does not seem to be a transient symptom, but rather an irreversible and slowly progressive symptom which often continues even after stopping such misuse. Based on the above findings, we stress that, in medically intractable cases, toluene induced tremors should be included in the positive indications for a Vim thalamotomy, along with other types of tremors.

1 Grabski DA. Toluene sniffing producing cerebellar degeneration. Am f Psychiatry 1961;118:461-2.
2 Kelly CTW. Prolonged cerebellar dysfunction associated with paint sniffing. Pediatrics 1975;56:605-6.

3 Metrick SA, Brenner RP. Abnormal brainstem auditory voked potentials in chronic paint sniffers. Ann Neurol 1982;12:553-6.

4 Rosenberg NL, Kleinschmidt-DeMasters BK, Davis KA, et al. Toluene abuse causes diffuse central nervous system white matter changes. Ann Neurol 1988;23:611-4.

5 Hirai $\mathrm{H}$, Ikeuchi Y. MRI of chronic toluene intoxication. Clin Neurol 1993;33:552-5.

6 Kojima S, Hirayama K, Furumoto H, et al. Magnetic resonance imaging in chronic toluene abuse, and volitional hyperkinesia. Clin Neurol 1993;33:477-82.

7 Sodeyama N, Wakayama Y, Takada $\mathrm{H}$, et al. A case of chronic thinner intoxication developing hyperkinesie volitionelle three years after stopping thinner abuse. Clin Neurol 1993;33:213-15.

8 Suzuki K, Orimo S, Okiyama R, et al. A case of chronic toluene intoxication with abnormal MRI findings: abnor-
mal intensity areas in cerebral white matter, basal ganglia, internal capsule, brainstem and middle cerebellar peduncle. Clin Neurol 1992;32:84-7.

9 Samie MR, Selhorst JB, Koller WC. Post-traumatic midbrain tremors. Neurol 1990;40:62-6.

10 Ohye C. Neural circuits involved in Parkinsonian motor disturbance studied in monkeys. Eur Neurol 1987;26(suppl $1): 41-6$.

11 Biary N, Cleeves L, Findley LJ, et al. Post-traumatic tremor. Neurol 1989;39:103-6.

\section{HISTORICAL NOTE}

\section{Post-traumatic meningioma: case report and historical perspective}

We report the case of 63 year old man found to have a calcified right frontal mass at the site of an 11 year old skull fracture. We present this case in support of the origin of some meningiomas from post-traumatic head injuries. In this case, the point of injury was precisely the site from which the tumour was removed and correlated directly with the traumatic injury to the inner table of the calvarium.

It is of no small consequence that Cushing felt strongly that meningiomas were caused by trauma to the calvarium with resultant meningeal irritation. The well published cases of some of his patients correlated the occurrence of these tumours with prior trauma to the skull and meninges. ${ }^{1}$ This association was highlighted by the well publicised case of General Leonard Wood and the patient's first craniotomy performed by Cushing in $1909 .{ }^{12}$ The successful removal of a "a brain tumour" on an eminent, public figure ensconced neurosurgery in the public's eye as a specialty in which surgery could be performed safely on the brain and people could survive. ${ }^{3}$ It was Dr Cushing's success that helped raise neurosurgery to new and distinguished heights and enabled him to become one of the most outstanding men in medical history.

This 63 year old black man presented to the hospital after a witnessed grand mal seizure. Eleven years earlier he had had a focal skull injury to the right frontal bone with an open laceration after being struck by a full beer bottle while on a fishing trip. Physical examination disclosed only a mild asymmetric motor weakness in the left arm and leg. Skull radiography showed a right frontal bone lytic lesion about 2 $\mathrm{cm}$ in diameter, hyperostosis, and an extraaxial calcified density (ill defined) directly beneath the lesion. Head CT disclosed a right frontal mass with a lytic focus involving the inner table.

A bicoronal incision and right frontal craniotomy was performed. A right frontal meningioma was found, producing full thickness skull erosion associated with a healed depressed skull fracture. The meningioma was resected with adherent dura and then sent for frozen section. The dura was closed primarily and a cranioplasty was performed using titanium mesh and methylmethalcrylate. Histopathological examination confirmed the diagnosis of meningioma (figure).

This case lends credence to the monumental work of Cushing over 75 years ago, who noted multiple cases of tumour formation after blunt or sharp head trauma. He cited 24 cases "in which evidence of an injury in the nature of a swelling, cicatrix, or depressed fracture corresponded with the tumor's obvious point of origin". Cushing noted that trauma as "an aetiological factor...is inescapable". ${ }^{4}$ The celebrated case of General Leonard Wood highlighted Continued on page 798 